\section{mutY 'directs' mutation?}

SIR - The ability of cells to undergo spontaneous mutation while in the resting state was demonstrated by Ryan et al. with Escherichia coli more than 30 years ago, but received little attention until the report by Cairns and collaborators ${ }^{2}$ that the mutations that arose were 'directed', that is, they specifically overcame whatever starvation was imposed to maintain them in a non-growing state.

Apparently, 'directed' mutation to prototrophy is readily demonstrated in certain ochre auxotrophs starved of their require-

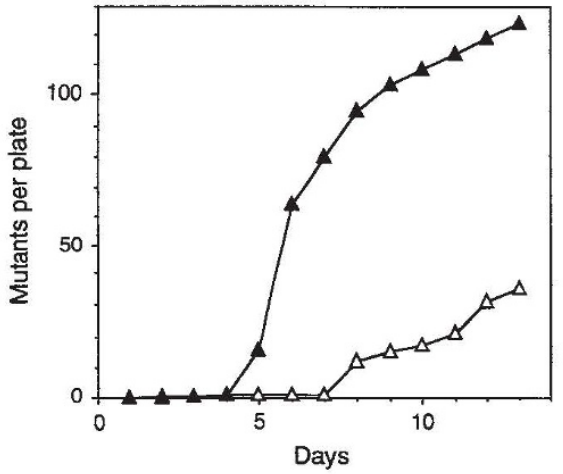

Appearance of tyrosine-independent mutants of bacteria incubated on plates lacking a required amino acid: influence of mutY. Bacteria were incubated overnight on oxoid nutrient broth no. 2 , centrifuged, resuspended in buffer and plated on minimal agar plates supplemented with $0.4 \%$ glucose and $10 \mu \mathrm{g} \mathrm{ml}^{-1}$ leucine, WU3610 tyrA14 $(\Delta)$ and its mutY68::kan ${ }^{R}$ derivative CM1321 (A) (initial inoculum $10^{8}$ bacteria per plate) (means of at least 3 experiments).

ments. The prototrophs that arise are slow-growing suppressors inferred to have arisen by transversion at $\mathrm{G}: \mathrm{C}$ base pairs and different from those commonly found to arise in growing bacteria ${ }^{3}$. This specificity, coupled with the time-dependent manner in which mutations arise on starvation plates, led to the suggestion that they are due to a DNA lesion. The lack of effect of DNA repair mutations such as $\operatorname{rec} A, u m u C, \operatorname{lex} A$ and $u v r A$ implied that this hypothetical lesion is a non-bulky type of damage generating mutations by miscoding rather than error-prone repair ${ }^{3}$.

A candidate for the hypothetical lesion in DNA is 7,8-dihydro-8-oxoguanine (8oxo-G), as this is known to cause $\mathrm{G}: \mathrm{C}$ to T:A transversions by incorporation of adenine opposite it, and is found in detectable quantities in cellular DNA. To mitigate its effect, bacteria have developed several enzymes including MutY, a glycosylase that removes adenine residues erroneously incorporated in DNA opposite 8-oxo-G.

Introduction of $m u t Y$ deficiency into the tyrA14 ochre strain WU3610 resulted in a dramatic increase in starvation-associated mutants (see figure). The excess mutants were slow-growing suppressors similar to those observed in the mut $Y^{+}$strain, showing that they can, as predicted, be caused by 8 -oxo-G. If the suppressor mutants that arise in the tyrA14 ochre strain under starvation conditions are at the anticodon of a transfer RNA locus, then the 8-oxo-G can be formed on the transcribed strand. Miscoding during transcription would result in a transient $\mathrm{Tyr}^{+}$phenotype, which could allow a round of DNA replication to occur. This round could permanently fix the mutation by a DNA mispairing event.

Such a mechanism could explain why starvation-associated mutation gives the appearance of being 'directed' in these strains. DNA replication is needed to fix the mutation by a mispairing event opposite 8 -oxo-G, but is inhibited by the restriction of protein synthesis due to the amino-acid auxotrophy. Only genes where mispairing opposite 8-oxo-G during transcription can give rise to a transiently prototrophic phenotype will ever have the opportunity to mispair during DNA replication.

It is not impossible that an analogous situation could exist in nondividing somatic cells of higher organisms. One might envisage a proto-oncogene that could trigger a cell replication cycle if a mispair occurred opposite an 8-oxo-G residue and if the 8oxo-G were in the transcribed strand. In the TP53 gene, for example, among more than 350 independent point mutations found in a wide variety of human cancers, there were 11 transversions where a guanine residue was in the transcribed strand ${ }^{4}$.

The hypothesis of transcriptional miscoding by 8 -oxo- $\mathrm{G}$ provides a plausible explanation of the extent of starvationassociated mutation in $\mathrm{mut}^{+}$cells and its apparently 'directed' nature. Although this hypothesis is not expected to explain all observations of 'directed' mutation, transversions at $\mathrm{G}: \mathrm{C}$ sites where the $\mathrm{G}$ is in the transcribed strand were by far the most common base-pair substitutions in a study of starvation-associated mutation with the set of lacZ strains of Cupples and Miller'. A similar phenomenon could in principle occur with other miscoding lesions in mutation systems that could respond to lesions on the transcribed strand. The data of Mackay et al. ${ }^{6}$ implicating $O^{6}$-alkylguanine in the induction of $\mathrm{G}: \mathrm{C}$ to $\mathrm{A}: \mathrm{T}$ transitions in stationary phase cells are entirely consistent with this interpretation.

\section{Bryn A. Bridges}

MRC Cell Mutation Unit,

University of Sussex,

Falmer, Brighton BN1 9RR, UK

1. Ryan, F. J. et al. J. gen. Microbiol. 30, 193-199 (1963).

2. Cairns, J. et al. Nature 335, 142-145 (1988).

3. Bridges, B. A. Mutat. Res. 307, 149-156 (1994)

4. Caron de Fromental, C. \& Soussi, T. Genes Chrom. Cancer 4, 1-15 (1992).

5. Hall, B. G. Genetica 84, 73-76 (1991)

6. Mackay, W. J. et al. J. Bact. 176, 3224-3230 (1994).
Life at extremely low pH

SIR - Only four organisms, oddly enough all eukaryotes, have so far been reported to grow at $\mathrm{pH}$ values around zero: the thermoacidophilic coccoid rhodophyte Cyanidium caldarium ${ }^{1}$; the fungi Acontium cylatium; Cephalosporium sp.'; and Trichosporon cerebriae ${ }^{3}$. We have now identified two related species of archaea from Japanese solfataras which can grow at and slightly below $\mathrm{pH} 0$.

The most acidophilic prokaryote previously described is the archaeal mycoplasm Thermoplasma acidophilum, which barely grows at $\mathrm{pH} 0.4$ and optimally thrives between $\mathrm{pH} 1.8$ and 2 (ref. 4). We have found two species of a novel genus of coccoid, hyperacidophilic, moderately thermophilic archaea, Picrophilus oshimae and Picrophilus torridus (DSM numbers 9789 and 9790, respectively), in samples from moderately hot (about $60{ }^{\circ} \mathrm{C}$ ) but extremely acidic places, a situation frequently encountered in solfataric fields. The organisms were isolated as single colonies on starch plates, both clearly growing around $\mathrm{pH} 0$ (the lowest $\mathrm{pH}$ was $-0.06 \pm 0.01$ measured at $20^{\circ} \mathrm{C}$ ) and optimally at $\mathrm{pH} 0.7$ (Fig. 1), the lowest $\mathrm{pH}$ optimum known to date. The optimal growth temperature was $60^{\circ} \mathrm{C}$. Both organisms were aerobic, nonfermenting heterotrophs, utilizing yeast extract and unable to grow by sulphur oxidation and reduction, or by sulphur respiration.

Phylogenetically, the closest relative of Picrophilus is T. acidophilum. The $16 \mathrm{~S}$ ribosomal RNA of $P$. oshimae (GenBank/EMBL accession number 84901) shows $90.7 \%$ sequence identity with that

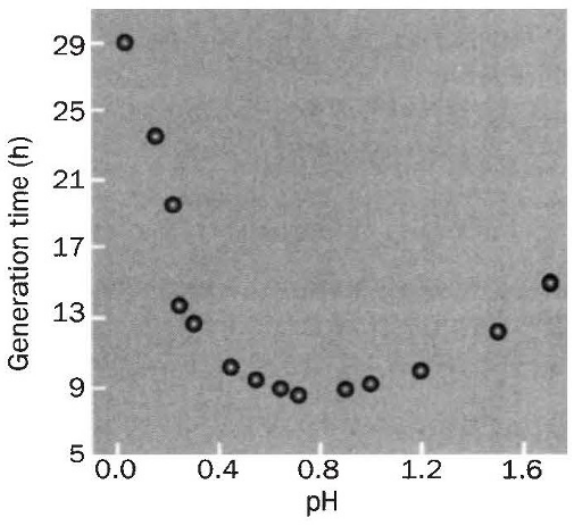

FIG. 1 Optimal pH of $P$. oshimae, isolate $\mathrm{KAW} 2 / 2$. Doubling times were calculated from the slopes of growth curves (not shown). The medium (according to E. A. Freundt ${ }^{7}$ ) contained $0.2 \%$ yeast extract as carbon source. $1 \mathrm{ml}$ each of late-logarithmic cultures grown under optimal conditions $\left(60^{\circ} \mathrm{C}\right.$ and $\mathrm{pH} 0.7$, aeration by moderate shaking) was used to inoculate $50 \mathrm{ml}$ of preheated growth media of varying $\mathrm{pH}$, measured with an Ingold Mettlerelectrode (type 405-60-S7/120 for $\mathrm{pH} \mathrm{O}$ to 12 ). $1 \mathrm{M}$ $\mathrm{HCl}$ was used as a reference for $\mathrm{pH} O$. 